FSU-CSIT-00-27

JLAB-THY-00-34

\title{
Exact Chiral Symmetry for Domain Wall Fermions with Finite $L_{s}{ }^{*}$
}

\author{
Robert G. Edwards ${ }^{\mathrm{a}}$ and Urs M. Heller ${ }^{\mathrm{b}}$ \\ a Jefferson Lab, 12000 Jefferson Avenue, MS 12H2, Newport News, VA 23606, USA \\ ${ }^{\mathrm{b}}$ CSIT, Florida State University, Tallahassee, FL 32306-4120, USA
}

\begin{abstract}
We show how the standard domain wall action can be simply modified to allow arbitrarily exact chiral symmetry at finite fifth dimensional extent $L_{s}$. We note that the method can be used for both quenched and dynamical calculations. We test the method using smooth and thermalized gauge field configurations. We also make comparisons of the performance (cost) of the domain wall operator for spectroscopy compared to other methods such as the overlap-Dirac operator and find both methods are comparable in cost.
\end{abstract}

\section{The problem with small eigenvalues}

Recently a great deal of theoretical progress has been made in the construction of lattice regularizations of fermions with good chiral properties 1.2]. For use in practical numerical simulations, though, approximations to these formulations are necessary. In the formulation using domain wall fermions (DWF) [1, 3], the extent of an auxiliary fifth dimension has to be kept finite in numerical simulations while chiral symmetry holds strictly only in the limit of infinite fifth dimension. The violations of chiral symmetry are expected to be suppressed exponentially in the extent of the fifth dimension [3. 4 , but in practice the coefficient in the exponent can be quite small [5.6] and the suppression correspondingly slow.

In the case of overlap fermions there is no such problem in principle. However, there is a problem of practicality: how to deal efficiently with $\epsilon(H)=H / \sqrt{H^{2}}$, where $H$ is some auxiliary Hermitian lattice Dirac operator for large negative mass, but free of doublers. Most commonly, the Hermitian Wilson-Dirac operator $H_{w}$ is used.

Any good numerical approximation to $\epsilon(H)$ must retain the property $\epsilon(H)^{2}=1$ which is crucial for the Ginsparg-Wilson relation

$\left\{\gamma_{5}, D_{o v}(0)\right\}=2 D_{o v}(0) \gamma_{5} D_{o v}(0)$

to hold and exact chiral symmetry for the overlap

*Presented by U. M. Heller at Lattice 2000
Dirac operator [7]

$D_{o v}(m)=\frac{1}{2}\left[1+m+(1-m) \gamma_{5} \epsilon(H)\right]$

at $m=0$ to be preserved. A good approximation of $\epsilon(x)$ is difficult to achieve for small $x$.

Unfortunately, at least in quenched simulations with the Wilson gauge action, $H_{w}(M)=$ $\gamma_{5} D_{w}(-M)$, with $D_{w}(M)$ the Wilson-Dirac operator, has a non-vanishing density of zero eigenvalues, $\rho(0 ; M)$, for any gauge coupling $\beta[8]$. $\rho(0 ; M)$ decreases quite rapidly with increasing $\beta$, roughly as $\rho(0 ; M) / \sigma^{3 / 2} \sim e^{-e^{\beta}}[\beta]$, but at currently used couplings a considerable number of modes with small eigenvalues exists.

In all numerical methods to implement overlap fermions one can enforce accuracy of the approximation to $\epsilon\left(H_{w}\right)$ by projecting out the lowest few $H_{w}$ eigenvectors and adding their correct contribution exactly (see Ref. [9]): with $H_{w} v_{i}=\lambda_{i} v_{i}$,

$$
\begin{aligned}
\epsilon\left(H_{w}\right) & =\sum_{i=1}^{n}\left|v_{i}\right\rangle \epsilon\left(\lambda_{i}\right)\left\langle v_{i}\right|+\mathcal{P}_{\perp}^{(n)} \operatorname{App}\left[\epsilon\left(H_{w}\right)\right] \mathcal{P}_{\perp}^{(n)}, \\
\mathcal{P}_{\perp}^{(n)} & =\mathbf{1}-\sum_{i=1}^{n}\left|v_{i}\right\rangle\left\langle v_{i}\right| .
\end{aligned}
$$

The number $n$ of projected eigenvectors is chosen such that the approximation $\operatorname{App}\left[\epsilon\left(H_{w}\right)\right]$ to $\epsilon\left(H_{w}\right)$ is sufficiently accurate in the subspace spanned by $\mathcal{P}_{\perp}^{(n)}$. 


\section{Implications for domain wall fermions}

Integrating out the heavy fermions, one can show that the effective Dirac operator for the physical 4-d fermions from the domain wall fermion approach is 10,11.

$D_{\text {tov }}(m)=\frac{1}{2}\left[1+m+(1-m) \gamma_{5} \varepsilon_{L_{s} / 2}\left(H_{T}\right)\right]$

with $\varepsilon_{N}(x)$ the polar decomposition approximation to $\epsilon(x)[12$

$$
\begin{aligned}
\varepsilon_{N}(x) & =\frac{(1+x)^{2 N}-(1-x)^{2 N}}{(1+x)^{2 N}+(1-x)^{2 N}} \\
& \approx \tanh (2 N x)
\end{aligned}
$$

and 13

$H_{T}(M)=H_{w}(M) \frac{1}{2+\gamma_{5} H_{w}(M)}$.

Zero modes of $H_{w}(M)$ are also zero modes of $H_{T}(M)$ !

Small eigenvalues of $H_{T}$ induce chiral symmetry violations, unless $L_{s}$ is very large $-\tanh (y)$ is within about $10^{-5}$ of 1 for $y \geq 6.1$. And the density of small eigenvalues of $H_{T}$ is the same as the density of small eigenvalues of $H_{w}$ !

Manifestations of the induced chiral symmetry breaking are, for example, violations of the Gellmann-Oakes-Renner relation

$m \chi_{\pi} \equiv m\left\langle\sum_{x} \pi(x) \pi(0)\right\rangle=\langle\bar{\psi} \psi\rangle$,

finding a non-zero pion mass for massless quarks, and absence of exact zero modes in topologically non-trivial gauge field backgrounds. This latter is illustrated in Fig. 11 where we show the spectral flow of the hermitian domain wall operator $H_{D W}(0 ; M)=\gamma_{5} \mathcal{J} D_{D W}^{(5)}(0 ; M)$, with $\mathcal{J}$ the inversion operator of the fifth direction, the hermitian Wilson operator $H_{w}(M)$ and the hermitian overlap $H_{o v}(0 ; M)=\gamma_{5} D_{o v}(0: M)$ on a single instanton configuration. Right after a Wilson eigenvalue crosses zero the overlap operator has an exact zero mode while the lowest eigenvalue of the domain wall operator is clearly non-zero even for $L_{s}=32$ for $M$ considerably after the crossing.

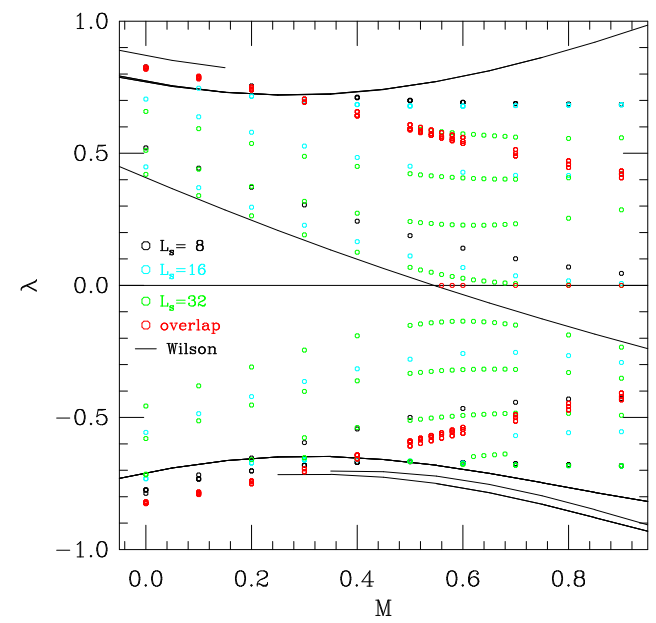

Figure 1. Eigenvalues of the hermitian domain wall, Wilson and overlap operators as function of the negative Wilson mass value $M$ on a single instanton configuration.

\section{Exact chiral symmetry at finite $L_{s}$}

With finite lattice spacing in the $5^{\text {th }}$ direction, $a_{5}$, the domain wall fermion (DWF) action is

$S_{D W}=-\bar{\Psi} D_{D W}^{(5)} \Psi$

The kernel of the 5-d operator is, including the boundary conditions used to introduce an explicit quark mass $m$,

$$
\begin{aligned}
& D_{D W}^{(5)}= \\
& \left(\begin{array}{ccccc}
D_{+}-\hat{A} P_{-} & -P_{-} & \cdots & 0 & (m-\hat{A}) P_{+} \\
-P_{+} & D_{+} & \cdots & 0 & 0 \\
\vdots & \vdots & \vdots & \vdots & \vdots \\
0 & 0 & \cdots & D_{+} & -P_{-} \\
m P_{-} & 0 & \cdots & -P_{+} & D_{+}
\end{array}\right)
\end{aligned}
$$

where $P_{ \pm}=\left(1 \pm \gamma_{5}\right) / 2$ and $D_{+}=a_{5} D_{w}(-M)+1$. $\hat{A}(m)$ is the new ingredient, acting on the light fermions, chosen to insure chiral symmetry. We integrate out the $L_{s}-1$ extra fermion fields and 
the pseudo-fermions to obtain [1]

$$
\begin{aligned}
& D_{\text {tov }}\left(m ; H_{T}\right) \\
= & \left\{\mathcal{P}^{-1}\left[D_{D W}^{(5)}(1)\right]^{-1} D_{D W}^{(5)}(m) \mathcal{P}\right\}_{11} \\
= & \frac{1}{2}\left[1+m+(1-m) \gamma_{5} \varepsilon_{L_{s} / 2}\left(a_{5} H_{T}\right)\right] \\
& + \text { term with } \hat{A}(m) .
\end{aligned}
$$

Here $\varepsilon_{N}(x)$ is the polar decomposition approximation to $\epsilon(x)$ introduced in (5), and $\mathcal{P}$ is such that $\left(\mathcal{P}^{-1} \Psi\right)_{1}=q$ is the light fermion field,

$\mathcal{P}_{j k}=\left\{\begin{array}{ll}P_{-} \delta_{j, k}+P_{+} \delta_{j+1, k} & \text { for } j<L_{s} \\ P_{-} \delta_{L_{s}, k}+P_{+} \delta_{1, k} & \text { for } j=L_{s}\end{array}\right.$.

$H_{T}$, given in $(6)$, is the auxiliary Hamiltonian associated with the transfer matrix $T$ in the $5^{\text {th }}$ direction,

$T \equiv \frac{1-a_{5} H_{T}}{1+a_{5} H_{T}}$.

With $H_{T} v_{i}=\lambda_{i} v_{i}, T v_{i}=T_{i} v_{i}$ we set,

$$
\begin{aligned}
& \hat{A}(m)=(1-m) \gamma_{5}\left(H_{w} P_{-}-1\right) \sum_{i=1}^{n} g_{i}\left|v_{i}\right\rangle\left\langle v_{i}\right| \\
& g_{i}=\frac{1}{2}\left[-\left(T_{i}^{-L_{s}}-1\right)+\left(T_{i}^{-L_{s}}+1\right) \epsilon\left(\lambda_{i}\right)\right]
\end{aligned}
$$

This "projects" low-lying eigenvalues of $H_{T}$ from $\varepsilon_{L_{s} / 2}\left(a_{5} H_{T}\right)$ and gives the correct $\epsilon\left(\lambda_{i}\right)$. Then $D_{\text {tov }}\left(m ; H_{T}\right)$ becomes $D_{o v}\left(m ; H_{T}\right)$,

$D_{o v}\left(m ; H_{T}\right)=\frac{1}{2}\left[1+m+(1-m) \gamma_{5} \epsilon\left(H_{T}\right)\right]$

the overlap operator with auxiliary Hamiltonian $H_{T}$.

With projection of the 5 lowest eigenvectors of $H_{T}$ we now find the expected zero-modes of the massless projected domain wall operator $\sqrt{\left(D_{D W}^{(5)}(0)\right)^{\dagger} D_{D W}^{(5)}(0)}$ for the same instanton configuration as in Fig. 1 already for moderate $L_{s}$, as can be seen in Fig. 2.

As already mentioned, the Gellmann-OakesRenner relation (7) is a sensitive test of chiral symmetry. For overlap fermions

$m\left\langle b\left|\left(\gamma_{5} \tilde{D}_{\text {ov }}^{-1}(m)\right)^{2}\right| b\right\rangle=\left\langle b\left|\tilde{D}_{\text {ov }}^{-1}(m)\right| b\right\rangle$

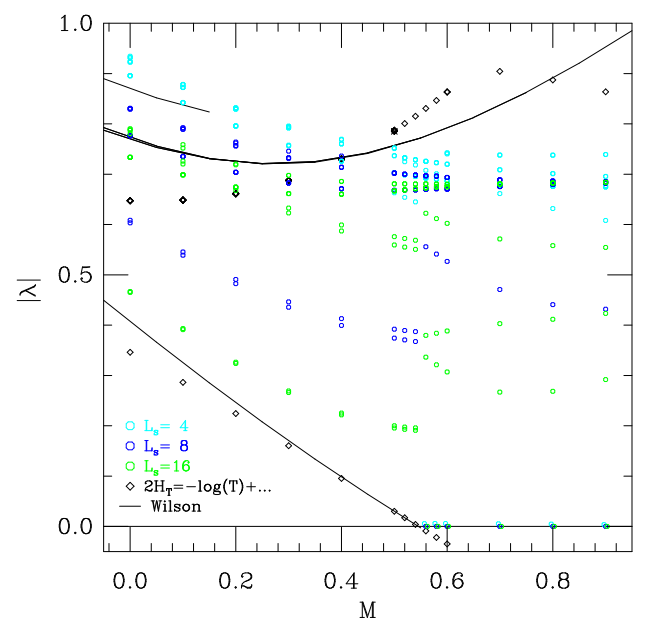

Figure 2. Eigenvalues of the domain wall operator with projection as function of the negative Wilson mass value $M$ on a single instanton configuration. Also show is the Wilson operator spectral flow and the eigenvalues of $2 H_{T}(M)$.

holds for any chiral vector $\gamma_{5}|b\rangle= \pm|b\rangle$, and averaging over several chiral Gaussian vectors $|b\rangle$ this becomes a stochastic estimate for the GellmannOakes-Renner relation (7). In (16) $\tilde{D}_{\text {ov }}^{-1}(m)$ is the external fermion propagator with the contact term subtracted and multiplicatively normalized [9], which avoids $\mathcal{O}(a)$ effects [14],

$$
\begin{aligned}
& (1-m) \tilde{D}_{o v}^{-1}(m)=\left[D_{o v}^{-1}(m)-1\right] \\
= & {\left[\left\{\mathcal{P}^{-1}\left[D_{D W}^{(5)}(m)\right]^{-1} D_{D W}^{(5)}(1) \mathcal{P}\right\}_{11}-1\right] . }
\end{aligned}
$$

We note that for standard DWF without projection the subtraction and multiplicative normalization is done automatically, i.e., with $\bar{q}=$ $(\bar{\Psi} \mathcal{J P})_{1}$ where $\mathcal{J}_{i j}=\delta_{i, L_{s}+1-j}$ is the inversion operator of the fifth direction,

$$
\begin{aligned}
\langle q \bar{q}\rangle & =\left\{\mathcal{P}^{-1}\left[D_{D W}^{(5)}(m)\right]^{-1} \mathcal{J P}\right\}_{11} \\
& =\frac{1}{1-m}\left[D_{\text {tov }}^{-1}(m)-1\right] .
\end{aligned}
$$

Finite $L_{s}$ and no projection lead to strong violations of the Gellmann-Oakes-Renner relation (7). With projection of a sufficient number of low eigenvectors the GMOR relation holds down to much lower quark masses, as seen in Fig. 3 . 


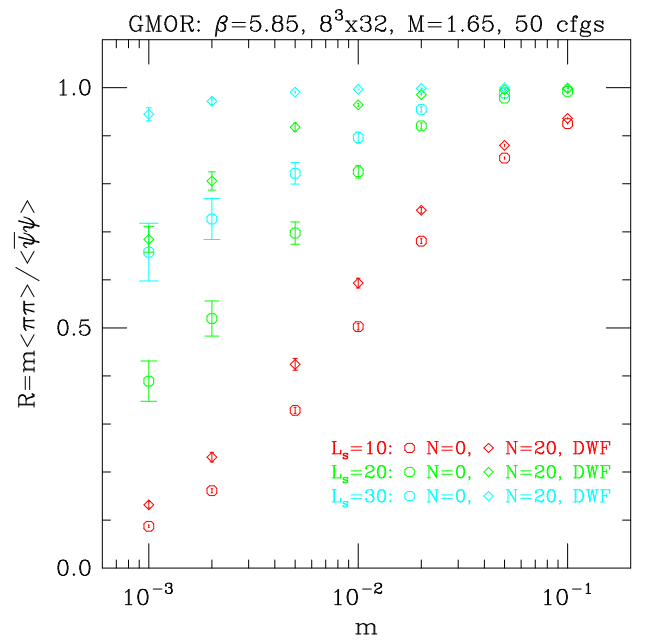

Figure 3. The ratio $R=m \chi_{\pi} /\langle\bar{\psi} \psi\rangle$ for DWF without and with projection of the lowest 20 eigenvectors.

\section{Comparing Efficiencies}

Having different possibilities for chiral fermion formulations on the lattice we can compare their efficiencies in numerical simulations. For this we consider a spectroscopy calculation for a topologically trivial gauge configuration. The cost is measured in the number of times $D_{w}$, common to all approaches, has to be applied on a 4 -d vector. This is shown in Fig. 囵.

For a single quark mass preconditioned DWF, with projection to assure minimal induced chiral symmetry breaking (see Fig. 3) at negligible cost, appears most efficient. However, in a quenched spectroscopy calculation with overlap fermions one can use a multi-shift inverter to compute for all quark masses simultaneously, compensating for the factor 3-4 in increased cost. Which regularization to prefer depends thus on the details of the numerical simulation attempted. If DWF are chosen then the projection method described here and in [11] should definitely be used to ensure good chiral properties at moderate $L_{s}$.

This research was supported in part by DOE contracts DE-AC05-84ER40150, DE-FG0596ER40979 and DE-FG02-97ER41022.

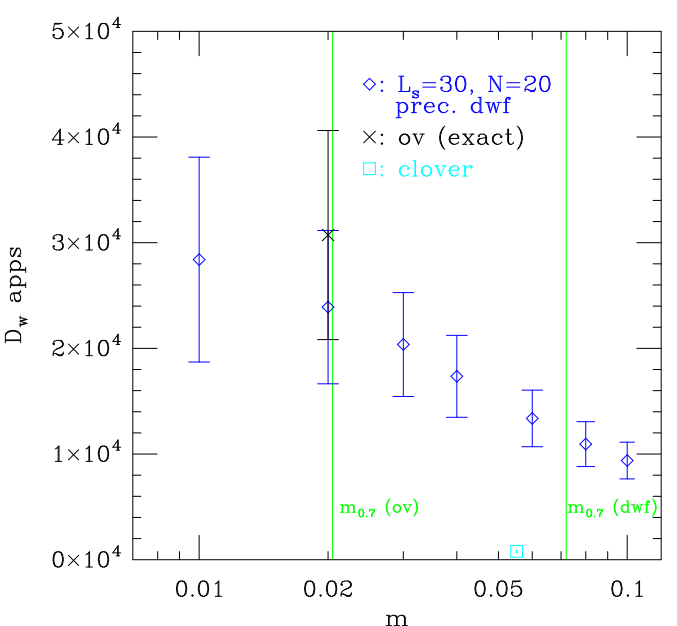

Figure 4. The cost of various implementations of chiral fermions as measured by the number of applications of $D_{w}$ on a 4 -d vector.

\section{REFERENCES}

1. D.B. Kaplan, Phys. Lett. B288 (1992) 342.

2. R. Narayanan and H. Neuberger, Nucl. Phys. B443 (1995) 305.

3. Y. Shamir, Nucl. Phys. B406 (1993) 90.

4. P. Vranas, Phys. Rev. D57 (1998) 1415; S. Aoki and Y. Taniguchi, Phys. Rev. D59 (1999) 054510; Y. Kikukawa and H. Neuberger, Nucl. Phys. B526 (1998) 572.

5. P. Chen et al., hep-lat/9812011; T. Blum, Nucl. Phys. Proc. Suppl. 73 (1999) 167.

6. G. Fleming, hep-lat/9909140; S. Aoki, T. Izubuchi, Y. Kuramashi, and Y. Taniguchi, hep-lat/0004003.

7. H. Neuberger, Phys. Lett. B417 (1998) 141.

8. R.G. Edwards, U.M. Heller and R. Narayanan, Phys. Rev. D60 (1999) 034502.

9. R.G. Edwards, U.M. Heller and R. Narayanan, Parallel Computing 25, 1395 (1999).

10. H. Neuberger, Phys. Rev. D57, 5417 (1998).

11. R.G. Edwards and U.M. Heller, heplat/0005002, to appear in Phys. Rev. D.

12. H. Neuberger, Phys. Rev. Lett. 81, 4060 (1998).

13. A. Boriçi, hep-lat/9912040.

14. S. Capitani et al., Phys. Lett. B468 (1999) 150. 